\title{
BUCHBESPRECHUNG
}

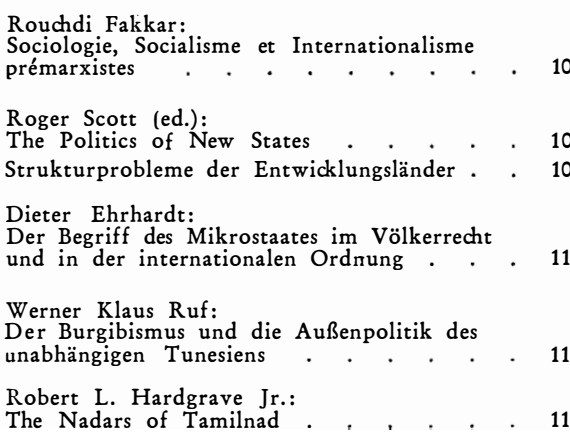

ROUCHDI FAKKAR

Sociologie, Socialisme et Internationalisme prémarxistes

L'influence de St. Simon - Neuchâtel 1968, 333 S.

Wenn alte und neue Staaten auf gleichem Fuße auch geistig miteinander kommunizieren sollten, dann ist damit gefordert, daß die Wissenschaft auch der neuen Staaten sich mit den politischen Ideen und ihren Autoren beschäftigt, die die alten Staaten hervorgebracht haben. Der Verfasser, Professor an der Universität von Rabat, leistet wohl als einer der Ersten einen Beitrag zur Befriedigung dieser nationalen und internationalen Notwendigkeit. Sein Werk ist eine ungemein sorgfältige und vollständige Darstellung der Ausbreitung, die die Ideen des Grafen St. Simon innerhalb und außerhalb Europas genommen haben. Für Übersee bemerkenswert ist hieraus vor allem die Wirksamkeit, die die St.-Simonisten, unter der Führung ihres Propheten Enfantin in Agypten entfaltet haben. Wie angesichts der Begeisterung ihres Meisters für Kanäle nicht anders zu erwarten, beschäftigten sie sich hier vor allem mit dem Plan einer Verbindung von Mittel- und Rotem Meer, und zwar so intensiv, daß man sie mit Fug zu den Vätern dieses Gedankens rechnen darf. Irgendeine Nachwirkung auf den heutigen "arabischen Sozialismus" scheint dieser Aufenthalt allerdings nicht gehabt zu haben.
Paul Isoart:

Le phénomène national viêtnamien . . . 114

Paul Isoart:

Le Viêtnam . . . . . . . . . 114

J. M. Mwanakatwe:

The Growth of Education in Zambia

since Independence. . . . . . . 118

David B. Abernethy:

The Political Dilemma of Popular Education 118

Martin C. Needler:

Political Systems of Latin America . . . 119

Gerhard Kutzner:

Die Organisation der Amerikanischen

Staaten (OAS). . . . .

ROger Scott (ed.)

The Politics of New States

A General Analysis with Case Studies from Eastern Asia

George Allen and Unwin, London 1970, $201 \mathrm{~S}$.

Das Buch ist aus einem der ersten undergraduate courses an australischen Universitäten hervorgegangen. Aus diesem Grunde sind die Beispiele, an denen die Politik der neuen Staaten dargestellt werden, aus dem Ostasiatischen Raum, der unmittelbaren Region Australiens, entnommen. In seiner Einleitung weist der Herausgeber zunächst mit Recht darauf hin, daß die Begriffe "new nations" und "developing areas" nicht genau genug das bezeichnen, was gemeinhin mit diesen Begriffen gemeint ist. Gerade das Nichtvorhandensein eines Nationalgefühls, das über die Ablehnung des colonialism hinausgeht, ist die Wurzel vieler Probleme der neuen Staaten. Der Begriff Entwicklungsländer paßt häufig besser auf die Staaten der Welt, deren Sozialprodukt sich wesentlich stärker entwickelt, als das der neuen Staaten. Geht man aber nicht von einem Wirtschaftswachstum, sondern von politischer und kultureller Entwicklung aus, so trägt man mit dem Begriff gleich den Anspruch an die Neuen Staaten heran, die politische und kulturelle Situation der Staaten der Alten Welt zu erreichen, obwohl keineswegs sicher ist, ob eine Entwicklung der Neuen Staaten in diese Richtung zu begrüßen wäre. 
Entwickeln soll sich jeder Staat, aber die Ziele muß er sich selber setzen. Der Herausgeber stellt dann die Hauptprobleme dar, die sich für die Politik in den neuen Staaten ergeben, wobei er besonders auf die Schwierigkeiten für die neuen Führer der Staaten eingeht, die sich aus der Tatsache ergeben, daß die zunächst an die Unabhängigkeit geknüpfte Hoffnung, es werde alles besser werden, nicht nur nicht erfüllt werden kann, sondern gemeinhin eher eine Verschlechterung der wirtschaftlichen Lage eingetreten ist. Er warnt davor zu glauben, die Neuen Staaten befänden sich nur auf einer frühen Stufe der Evolution und würden sich schließlich zum Standard westlicher Demokratie entwikkeln, denn die Umwelt und die Tradition sind so grundlegend anders, daß die Entwicklung notwendigerweise eine andere sein muß.

Die Länderbeiträge beginnen mit Japan und China, den beiden großen Antipoden im östlichen Asien. Beide sind zwar nicht wirklich Neue Staaten, weil beide nicht erst durch das Aufhören kolonialer Beherrschung zum Staat wurden, ja beide nie wirkliche Kolonien waren (China noch weniger als Japan), aber Japan zeigt einen Weg konsequenter Modernisierung, dem die politische $\mathrm{Re}-$ form nachgefolgt ist, während China das Vorbild für den Weg zur Modernisierung durch politische Revolution ist. Von beiden Staaten wird ein kurzer Abriß der Entwicklung (Japan seit 1868, China seit 1912) und der wesentlichsten Strömungen im politischen Leben gegeben. Es folgt dann ein Beitrag über die Auslandschinesen in Südostasien, die durch ihre starke kulturelle Bindung ans Mutterland das Problem der Konsolidierung, das durch die Vielfalt der Gruppen in den Neuen Staaten ohnehin schwierig genug ist, beträchtlich vergrößern. Dabei ist zu fragen, ob es überhaupt möglich ist, für einen wirtschaftlich und politisch noch schwach strukturierten Staat eine sich so einheitlich darstellende Gruppe mit starker wirtschaftlicher Macht $\mathrm{zu}$ integrieren. In Thailand scheint sich eine Integration abzuzeichnen, während in Malaysia die
Chance einer Integration verloren $z \mathbf{u}$ sein scheint.

Der folgende Beitrag über Vietnam gibt eine allgemeine Einführung in die politische Lage des Südens und stellt kurz die Politik Nordvietnams dar. Der Verfasser, der selbst in Vietnam gelebt hat, gibt eine gute Abwägung des Pro und Contra einer Entscheidung für die NLF oder das bestehende Regime und sieht wenig Aussicht, daß das bestehende Regime sich auf die Dauer wird gegen den Einfluß von Norden durchsetzen können. Langfristig sei mit einem Gesamtstaat Vietnam unter kommunistischer Führung zu rechnen.

Der Beitrag über Indonesien trägt den Untertitel ,The Archetypal New State'. In Indonesien zeigt sich die Entwicklung, vielleicht weil sie langsamer als anderswo vor sich ging, von der zunächst nach der Unabhängigkeit errichteten Demokratie, über die guided (unter Führung eines starken Mannes) democracy zum Militärregime beziehungsweise zur Herrschaft nur einer Gruppe besonders deutlich. Der Grund hierfür liegt in der Unfähigkeit der Parteien, eine gesamtstaatliche Politik zu betreiben, da sie nur von bestimmten sich von den anderen scharf unterscheidenden Volksgruppen getragen werden. Eine Entwicklung, die zum Beispiel nach der Aufsplitterung des Kongresses auch in Indien droht, da die regierende noch sich auf unterschiedliche Gruppen stützende Partei an Stärke in die Nähe der Interessenparteien gerät.

Ein letzter Beitrag vergleicht Neu Guinea und das tropische Afrika. Beide Regionen sind im Unterschied zu den anderen asiatischen Ländern nur sehr kurz wirklich kolonialisiert gewesen. Bei beiden Regionen hat sich der Wille zu Unabhängigkeit in wenigen Jahren entwickelt, ohne daß der neue Staat, der entsteht, auf andere entwickelte politische Strukturen zurückgreifen kann als die traditionellen Stammesordnungen. Das große Plus der asiatischen Staaten, eine lange Geschichte - viel länger als die der Kolonisation - als Kulturnation $\mathrm{zu}$ besitzen, fehlt in vielen afrikanischen Staaten und in Neu Guinea, 
wenn auch die Kulturleistungen dieser Regionen nicht unterschätzt werden dürfen. Aber in diesen Regionen beruht das gesamte Erziehungssystem auf der Mission, während die anderen asiatischen und ein Teil der afrikanischen $\mathrm{Na}$ tionen ein islamisches oder buddhistisches Erziehungssystem besaßen, das den westlichen Einfluß verarbeiten und überstehen konnte. Der starke Einfluß, den die traditionellen Gruppen auf den einzelnen haben, erschwert das Erwachsen eines gemeinsamen Nationalgefühls, das für die Errichtung eines größeren Staatsgebildes unerläßlich ist. In $\mathrm{Neu}$ Guinea wird versucht, durch Erziehung und durch Förderung der Mobilität eine Bevölkerung zu schaffen, die in der Lage ist, Probleme unter gesamtstaatlichen Aspekten zu lösen. Daneben wird versucht, durch Beteiligung der Bevölkerung an den politischen Entscheidungen, für die Erlangung der Unabhängigkeit eine Grundlage für die Formulierung der politischen Willensäußerungen $\mathrm{zu}$ schaffen. $\mathrm{Ob}$ in Neu-Guinea gelingt, was auch in Afrika erhofft wurde, dort aber durch die sich überstürzenden Ereignisse nicht mehr verwirklicht werden konnte, bleibt abzuwarten.

Der vorliegende Band gibt eine gute Einführung in die Probleme neuer Staaten besonders für den, der sich mit den Problemen im ostasiatischen Raum noch nicht beschäftigt hat. Die Kürze der Darstellung und die Weite des Themas führen sicher häufig zu Vereinfachungen, möglicherweise auch zu Verfälschungen, die Verfasser bemühen sich aber alle um eine möglichst neutrale unparteiische Darstellung, so $\mathrm{da} \beta$ das Buch Anfängern im Studium der Überseeischen Welt wärmstens empfohlen werden kann.

\section{Strukturprobleme der Entwicklungsländer}

hrsg. v. Institut für Internationale Solidarität der Konrad-Adenauer-Stiftung, Schriftenreihe Bd. 5

v. Hase und Koehler Verlag

Mainz 1969, 88 S.

Dieser vom Institut für Internationale Solidarität der Konrad-Adenauer-Stiftung herausgegebene Band mit vier Einzelbeiträgen soll, wie das Vorwort betont, mithelfen, eine Diskussionsgrundlage für die drängenden Probleme der Entwicklungsländer zu geben. Diesem Anspruch werden die Beiträge kaum gerecht.

Der erste Beitrag von Raupach, der das Verhältnis der Entwicklungsländer zwischen Ost und West behandelt, gibt außer einigen allgemein bekannten Argumenten für die Notwendigkeit von Entwicklungshilfe nur eine kurze Darstellung des Verhältnisses der Sowjetunion zu den Entwicklungsländern. Der Verfasser weist mit Recht darauf hin, daß die Größe der Probleme und die Schnelligkeit, mit der sie sich infolge des Bevölkerungswachstums zuspitzen, keine Zeit für lange politische Diskussionen lassen. Zur schwierigen Lage der Entwicklungsländer, die einerseits darauf achten müssen, keine der beiden Seiten durch $\mathrm{zu}$ offene Anlehnung an die andere $\mathrm{zu}$ verschrecken, andererseits aber häufig Hilfe nur unter politischen Vorbedingungen erhalten, nimmt der Verfasser keine Stellung.

Der zweite Beitrag von Dürr will Alternativen der Wachstumsplanung aufzeigen. Er bedient sich dazu der herkömmlichen wirtschaftspolitischen Modelle. Er lehnt eine direkte Wachstumsplanung ab mit dem Hinweis darauf, daß eine solche Planung außerordentlich schwierig sei, weil der marginale Kapitalkoeffizient kaum richtig prognostiziert werden könne. Darauf untersucht er die Planung der Wachstumsbedingungen. Er meint, daß unbedingte Voraussetzung eines Wachstums eine soziale Marktwirtschaft sei. Der Möglichkeit der Bildung eines Kapitalmonopols glaubt der Verfasser durch eine straffe 